\title{
Aaron's final program. A formal approach to the analysis of software archives for the social and historical sciences.
}

Camille Akmut

January 14, 2020

\begin{abstract}
A working version of keepgrabbing.py was reconstructed from archives.
\end{abstract}




\section{Software as archives}

In writing Aaron Swartz's JSTOR code we did not merely attempt a "technical" presentation, but sought to give a full "sociological, legal and historical" account - as part of our efforts to extend both the domain of one and the other science. These sciences meet now halfway, or they are the same.

These debates are far from settled.

Written over a period of a few weeks, though a long time in the coming ${ }^{1}$, in multiple iterations, we drew primarily from 3 documents :

- The source code of the program (entitled keepgrabbing.py).

- USA v. Swartz (hundreds if not thousands of pages of collected legal and technical prose ${ }^{2}$ )

— The Report to the President of MIT, ordered by the latter institution and written by computer scientist Hal Abelson inside of a larger team ${ }^{3}$.

Our efforts took us so far that we ended up reconstructing a working version;

From a careful analysis - as good as we could, anyway - followed chaotic consequences.

But, crucially, this reconstruction, while of strong scientific interest, was not able to accomplish anything with the inputs we gave to it, exempli gratia, the titles of two texts by Marc Bloch and Yitang Zhang.

Let alone that the infrastructure at JSTOR must have considerably changed since the last decade.

In any case, the moneyed interests of this institution cannot trump those of research, and the quest for knowledge and truth.

The main difficulty consisted in identifying the inputs passed to the lambda function (encapsulated in line) in the absence of the redacted passage of code.

But, this could be reversed engineered thanks to even summary knowledge of the Python standard library - as long as paired with a knowledge of functional programming.

The .split() function was key.

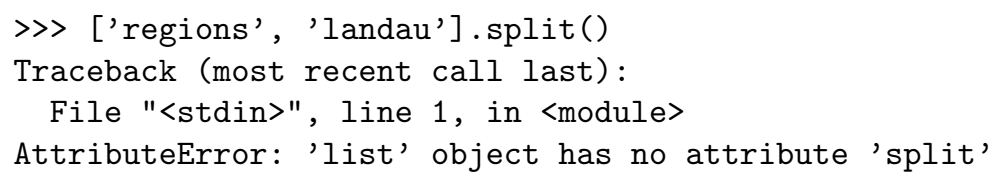

It only accepts certain types of objects. From there the rest could be derived.

\footnotetext{
${ }^{1}$ This research had for antecedent the relevant sections in "A User-Defined Web" (2019).

${ }^{2}$ We have made use of one document in particular : the so-called "Superseding indictment" from Sep. 2012. Others are encouraged to go through the rest - these inhumanely numerous pages are the new archives, of this new history and sociology.

${ }^{3}$ We presume it was Abelson who wrote, or made the biggest contributions to the more technical "Part 1" and connected apparatus (e.g. inventory of terms found at the end).
} 
The redacted passages point to a website with the ending /grab, of which we have presumed so far it was created by Aaron.

But, as we argue, even with no knowledge of this portion of the code, a black box, a correct or even partial understanding of the anonymous function lambda $\mathrm{x}$ was sufficient.

To be clear, keepgrabbing.py was not Aaron Swartz's last program ${ }^{4}$. But, it was the one that had the most effect in defining the next three years of his life, from 2010 to January 2013.

Aaron Swartz had lived in Cambridge, $\mathrm{MA}^{5}$, but moved away presumably as a result of the events, and hanged himself in Brooklyn, NY ${ }^{6}$.

We simplified the program, but did not lessen its functionalities :

Instead of receiving its inputs from the Internet/Web (via the Python module urllib), here it does so from a list, the same list it would have received, or close enough.

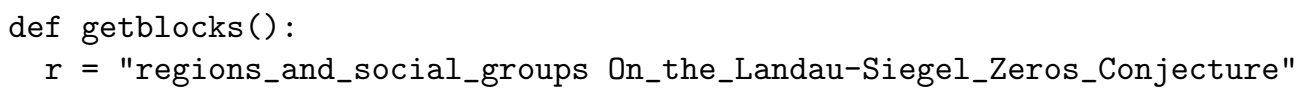

Simplying and experimenting, we cannot stress this enough, remain some of the most powerful tools in our equivalent of the archaeologist's toolkit.

A diagram to complete this discussion :

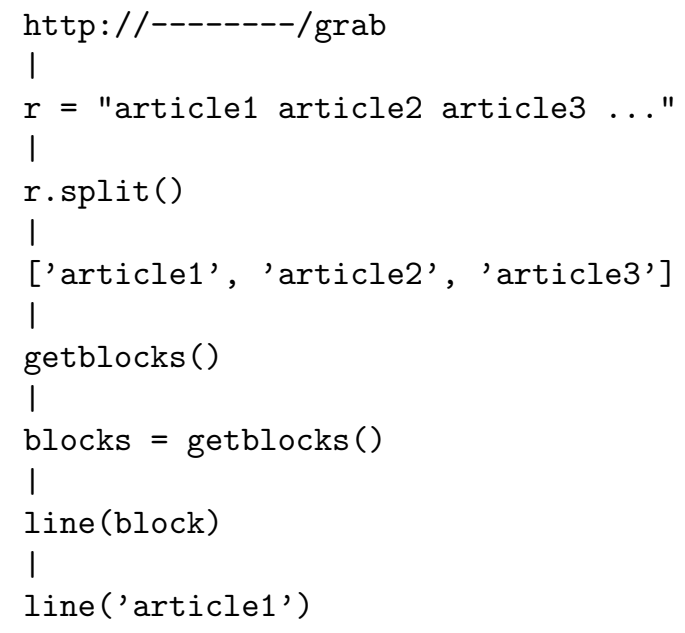

${ }^{4}$ See our history of his time spent on Github. ${ }^{5}$ See Abelson et al., beginning of part 2 of their report.

${ }^{6}$ His partner has given an account of their last days. 


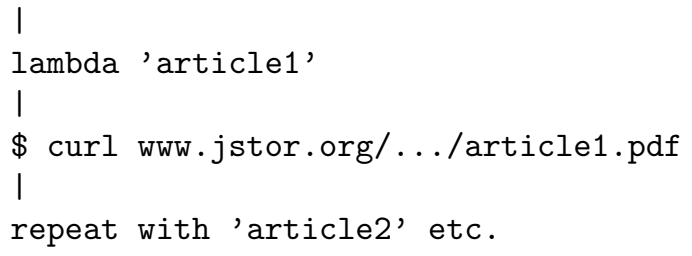

What follow are the last fragments of these experiments. 


\begin{abstract}
Annex
- 1 : raw code (as found about in archives i.e. the legal documents)

-2 : working version (the redacted part is bypassed as well as the use of proxy, we assume the simplest scenario) do not execute!

-3 : this version is run successfully for the first time (after multiple tries and modifications resulting in 2)

$-4:$ this version is run a second time by accident (inside the Python shell)

-5 : a second version for local testing is created

-6 : output of the second version : the $\mathrm{x}$ to the lambda function is as intended and the rest too
\end{abstract}


\#! /usr/bin/python

import subprocess, urllib, random

class NoBlocks(Exception): pass

def getblocks ():

$r=$ urllib.urlopen("http://- - - - - - /grab"). $\operatorname{read}()$

if '<html' in $r$.lower(): raise NoBlocks

return r.split()

import sys

if len(sys.argv) > 1:

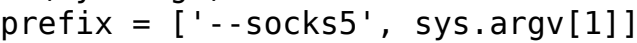

else:

prefix $=[]^{\prime}$ '-interface', 'etho: 1']

line = lambda $x:$ ['curl'] + prefix + ['-H', "Cookie: TENACIOUS $="+$

str (random. random())[3:], '-o', 'pdfs/' + str(x)+'.pdf', "http://www.jstor.org/

stable/pdfplus/" + str $(x)+$ ".pdf?acceptTC=true"]

while 1:

blocks = getblocks ()

for block in blocks:

print block

subprocess. Popen (line (block)) . wait( ) 


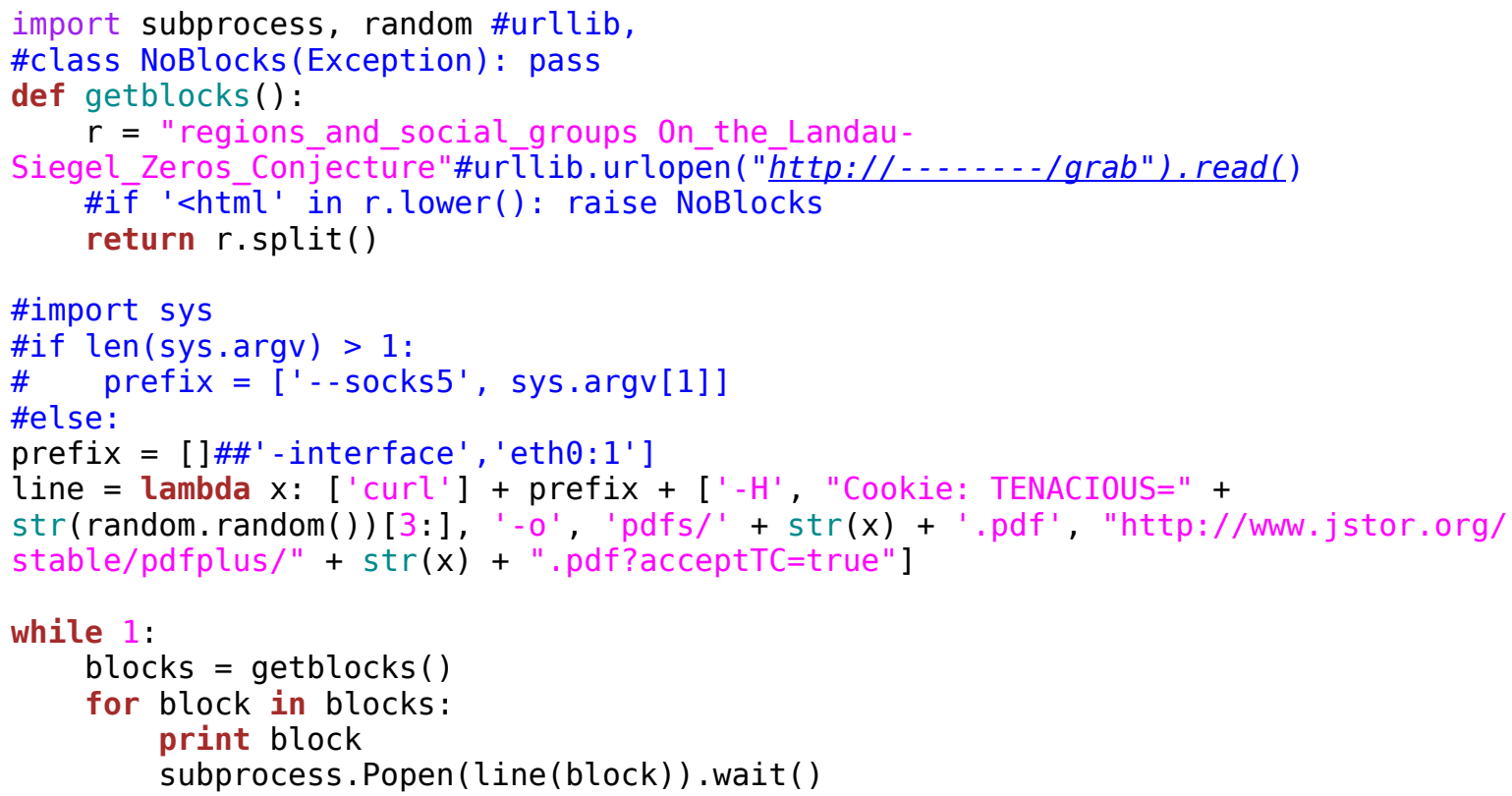




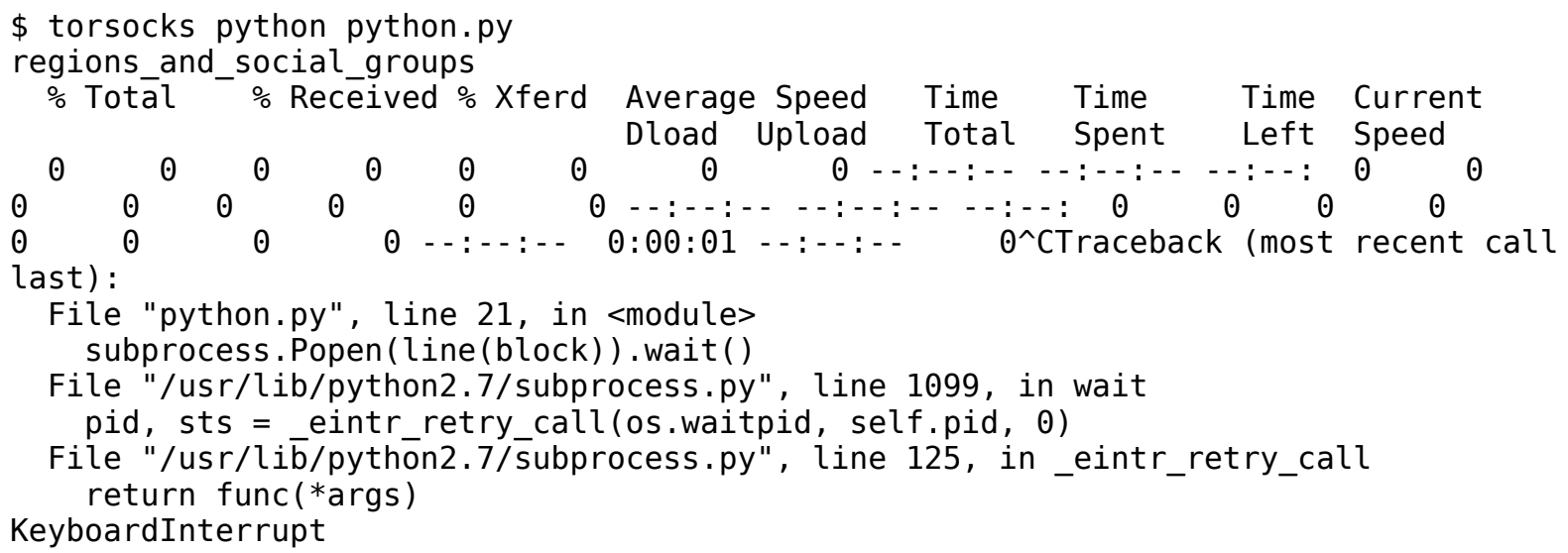


>> import python.py

regions and social groups

$\%$ Totăl $\%$ Received \% Xferd Average Speed Time Time Time Current

Dload Upload Total Spent Left Speed

OWarning: Failed to create the file pdfs/regions_and_social_groups.pdf: No such Warning: file or directory

$\begin{array}{lllllll}100 & 117 & 100 & 117 & 0 & 0 & 319\end{array}$

curl: (23) Failed writing body ( $0 \quad !=117)$

On_the_Landau-Siegel_Zeros_Conjecture

$\begin{array}{rllll}\text { \% Total \% Received \% } \bar{X} \text { ferd Average Speed Time Time } & \text { Time Current } \\ \text { Dload Upload Total Spent Left Speed }\end{array}$

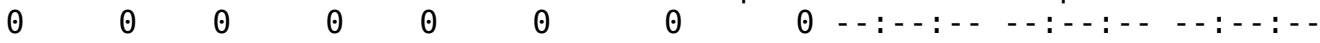

OWarning: Failed to create the file

Warning: pdfs/On_the_Landau-Siegel_Zeros_Conjecture.pdf: No such file or Warning: directory

$\begin{array}{lllllll}100 & 129 & 100 & 129 & 0 & 0 & 1535\end{array}$

○ - : : - : - - - : - : - - - : - : -

1535

curl: (23) Failed writing body ( $0 \quad !=129)$

regions_and_social_groups

$\%$ Totă - $\%$ Recéived \% Xferd Average Speed Time Time Time Current

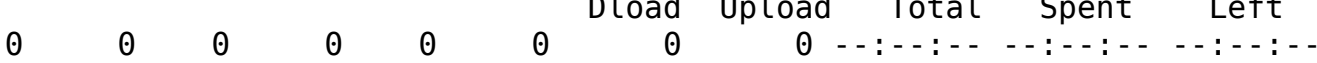

OWarning: Failed to create the file pdfs/regions_and_social_groups.pdf: No such Warning: file or directory

$\begin{array}{lllllll}100 & 117 & 100 & 117 & 0 & 0 & 1300\end{array}$

0 - - :- : - - - :- : : - - : - : - 1300

curl: (23) Failed writing body ( $0 \quad !=117)$

On the Landau-Siegel Zeros Conjecture

\% Total \% Received \% X́terd Average Speed Time Time Time Current

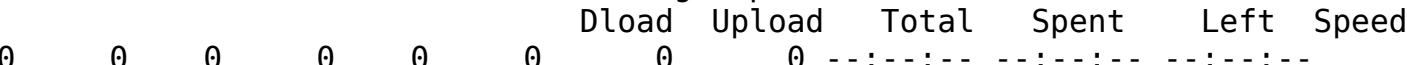

OWarning: Failed to create the file

Warning: pdfs/On_the_Landau-Siegel_Zeros_Conjecture.pdf: No such file or Warning: directory

$\begin{array}{lllllll}100 & 129 & 100 & 129 & 0 & 0 & 1402\end{array}$

○ - -:- : :- - : :-: - - - :- : - 1402

curl: (23) Failed writing body ( $0 \quad !=129$ )

regions and social groups

\% Totă $\%$ Recéived \% Xferd Average Speed Time Time Time Current $0 \quad 0 \quad$ Dload Upload Total Spent Left Speed

owarning: Failed to create the file pdfs/regions_and_social_groups.pdf: No such Warning: file or directory

$\begin{array}{lllllll}100 & 117 & 100 & 117 & 0 & 0 & 1026\end{array}$

0 - - : - : - - - : - : - - - : - : - 1026

curl: (23) Failed writing body ( $0 \quad !=117)$

On_the_Landau-Siegel_Zeros_Conjecture

\% Total \% Received \% X́Xferd Average Speed Time Time Time Current
0

$0 \quad 0 \quad 0 \quad 0 \quad 0$

OWarning: Failed to create the file

Warning: pdfs/On the Landau-Siegel_Zeros_Conjecture.pdf: No such file or Warning: directory

$\begin{array}{lllllll}100 & 129 & 100 & 129 & 0 & 0 & 1343\end{array}$

○--:--:-- - : - - :- - - :- - - -

1343

curl: (23) Failed writing body ( $0 \quad !=129)$

regions_and_social_groups

\% Totāl $\%$ Recéived \% Xferd Average Speed Time Time Time Current
0

$\begin{array}{rccc}\text { Dload Upload Total Spent Left } \\ 0 & 0 & \text { Sp - :-:-- - :--:- - - :--:- }\end{array}$

OWarning: Failed to create the file pdfs/regions_and_social_groups.pdf: No such Warning: file or directory

$\begin{array}{lllllll}100 & 117 & 100 & 117 & 0 & 0 & 1218\end{array}$

0 - - :- : - - - : - : : - - : - : - 1218

curl: (23) Failed writing body $(0 \quad !=117)$

On the Landau-Siegel Zeros Conjecture

$\overline{\%}$ Total \% Received \% X̄ferd Average Speed Time Time Time Current $\odot \quad 0 \quad 0 \quad 0 \quad$ Dload Upload Total Spent Left Speed 


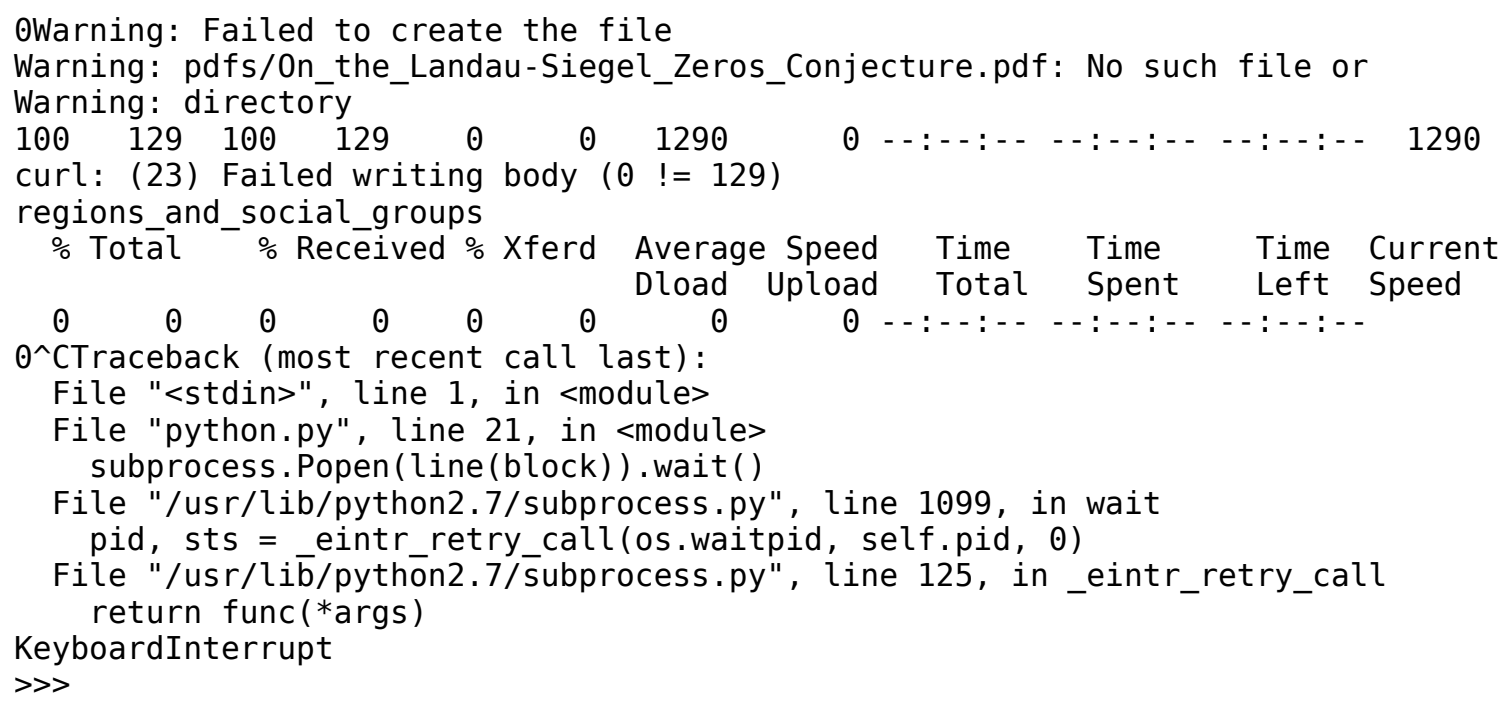




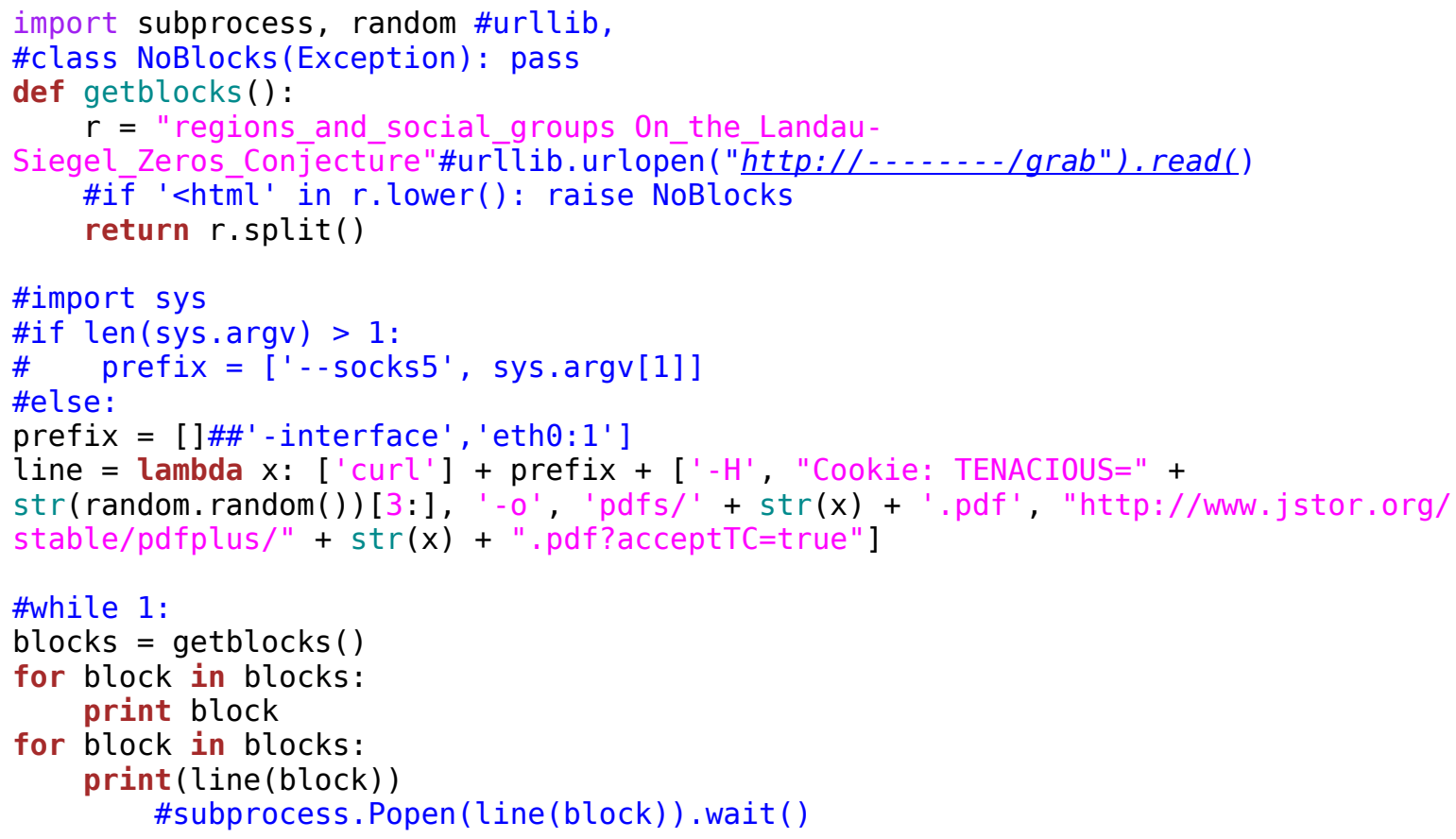


\$ python python2.py

regions and social_groups

On the Landāu-Siegē Zeros_Conjecture

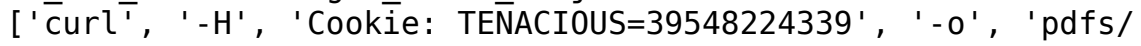

regions_and_social_groups.pdf', 'http://www.jstor.org/stable/pdfplus/

regions_and social_groups.pdf?acceptTC=true']

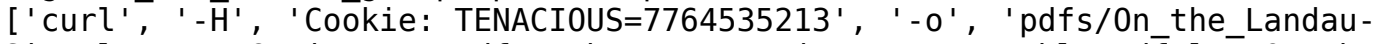

Siegel_Zeros_Conjecture.pdf', 'http://www.jstor.org/stable/pdf plus/On_the_Landau-

Siegel_Zeros_Conjecture.pdf?acceptTC=true'] 